\title{
A\&L
}

\section{The "Mix-Factor" is involved in the regulation of the organism's redox systems in the late stages of ontogenesis and affects the lifespan of animals}

\author{
Anatoly I. Bozhkov ${ }^{1}$, Ievgeniy G. Ivanov ${ }^{1}$, Elena M. Klimova ${ }^{2}$, Natalia I. Kurguzova ${ }^{1}$, Andrii A. Bozhkov ${ }^{1}$, \\ Anatoly V. Goltvyanskiy ${ }^{1}$, Yuri V. Nikitchenko ${ }^{1}$ \\ ${ }^{1}$ Research Institute of Biology, V.N. Karazin Kharkov National University, Kharkov, Ukraine; ${ }^{2}$ V. T. Zaitsev \\ Institute of General and Emergency Surgery of the NAMS of Ukraine, Kharkov, Ukraine \\ https://doi.org/10.47855/jal9020-2021-2-4
}

Correspondence: bozhkov@univer.kharkov.ua

Received: 16.07.2021; Accepted: 22.07.2021; Published: 03.08.2021

\begin{abstract}
: the content of lipid hydroperoxides in the serum and liver mitochondria in animals in the late stages of ontogenesis (12 and 33 months old, Wistar male rats) was investigated. The possible effect of the food additive ("mix factor") on these parameters in 33 months old animals, if they received this supplement from 22 months age daily throughout life in a dose of $0.05-0.06 \mathrm{ml} / 100 \mathrm{~g}$ of body weight was determined. Moreover, the lifespan of animals was determined if they started taking a mix - factor from 22 months and from 30.5 months age. The influence of the mix - factor on the ability of old animals to perform work was determined in a separate series of experiments. It was shown that from 22 to 33 months the content of lipid hydroperoxides in mitochondria and serum increased by $23 \%$ and $41 \%$, respectively. The activity of glutathione peroxidase in the serum, mitochondria and erythrocytes decreased, and the activity of glutathione reductase decreased only in the post-mitochondrial fraction of the liver. At the same time, malate- isocitrate dehydrogenase and 6-phosphate dehydrogenase remained unchanged in 33 months old animals compared with 12 months old animals. If animals from 22 months age received a mix factor with drinking water in a dose of 0.05-0.06 mg / $100 \mathrm{~g}$ of body weight every day until the end of life, the content of lipid hydroperoxides and the activity of glutathione peroxidase and glutathione reductase of 33 months old animals did not differ from 12 months old animals. The survival curve in animals receiving the mix factor was shifted to the right from 25 to 28 months of age compared with the survival curve of control animals. If animals began to receive the mix - factor at a very old age (from 30.5 months age) until the end of life, their lifespan was longer than that of the control animals. The last ones lived to 41 months, while the animals of the control group lived to 36 months. At the same time, the mix - factor increased the ability of old animals to do work in the test "running in treadmill".
\end{abstract}

Key words: aging, redox-system, geroprotectors, lifespan

Attempts to find or create means capable of increasing life expectancy were carried out long before the formation of gerontology as a science. Despite the advances in the study of the mechanisms of aging and the social significance of geriatric work, no significant results in this direction have yet been achieved. It is quite obvious that the development of geroprotectors should be based on knowledge of the aging mechanisms [1].

Numerous studies of possible mechanisms of ontogenetic changes made it possible to formulate several dozen hypotheses. Despite their diversity, the free radical hypothesis of aging remains the most discussed and, as before, completely unproven $[2,3,4,5]$. If the cause of age-dependent pathologies is an increase in the products of free radical reactions, then with a decrease in their content in old animals to the level of adult or young healthy animals, an increase in their lifespan could be expected. As a rule, hypothesis testing is based on the search for correlations between functional indicators (in particular, the prooxidantantioxidant system) and the life span of animals. This approach is also used in this work.

The study of indicators of the activity of the organism's redox system in ontogenesis is of interest not only from the standpoint of testing the free radical hypothesis but also because it is one of the ancient evolutionary systems of regulation in biological systems [6,7] and plays an important role in ontogenetic 
processes. Multiple attempts to regulate the redox system using various exogenous antioxidants have yielded conflicting results $[8,9]$.

We believed that such inconsistency and low efficiency of exogenous antioxidants can be explained by several reasons: 1 - high content of endogenous antioxidants, and against such a background exogenous antioxidants cannot have any significant effect; 2 - the products of free radical reactions perform important regulatory functions $[10,11]$ and the direction of their action depends on a large number of factors that can change in ontogenesis, or, in other words, one should not expect a direct correlation between the pro- and antioxidant system and life expectancy; 3 - aging is realized not according to one mechanism but can be realized in different ways, i.e. there are pronounced individual characteristics of the body's response to antioxidants.

Based on this, when developing geroprotectors, it is necessary to proceed from the fact that they will increase the life expectancy if:

1. will provide the regulation of not one, but several important functional systems of the body (redox system, immune system, detoxification system, etc.);

2. should improve the quality of life and increase its duration after intake, not at the early, but at the later stages of ontogenesis, i. e. after ingestion by adults and old animals.

In this regard, the search for new geroprotectors should include studies of the characteristics of the redox system as a basic regulatory system, and potential geroprotectors, at least, should ensure an increase in life expectancy at the later stages of ontogenesis.

Earlier it was shown that the developed polyligand lower molecular weight natural complex - "mixing factor" eliminated pathological manifestations that were induced by heavy metal ions $[12,13]$. The mixing factor took part in the regulation of the functions of the redox system [14,15], the immune system [16], and several physiological functions in experimental animals [12]. Moreover, the effect of the mixing factor was age-dependent [17].

In this regard, the content of lipid hydroperoxides and the activity of antioxidant enzymes was investigated: glutathione peroxidase, glutathione reductase, glucose - 6 - phosphate dehydrogenase, isocitrate dehydrogenase, and malate dehydrogenase in liver mitochondria and serum of adult animals, which took the mix factor daily from 22 months or 30.5 months until the end of their life. The influence of the mixing factor on the ability of old animals to perform work in the "treadmill running" test was determined.

\section{Materials and methods}

Experimental scheme for preparing animals for research

The experiments were carried out on adult (12 and 22 months old) and old ( 33 months old) male Wistar rats. The animals were kept under standard vivarium conditions. In the experiment on the effect of the mixing factor on the lifespan of adult animals, six groups of animals were formed (Fig. 1).

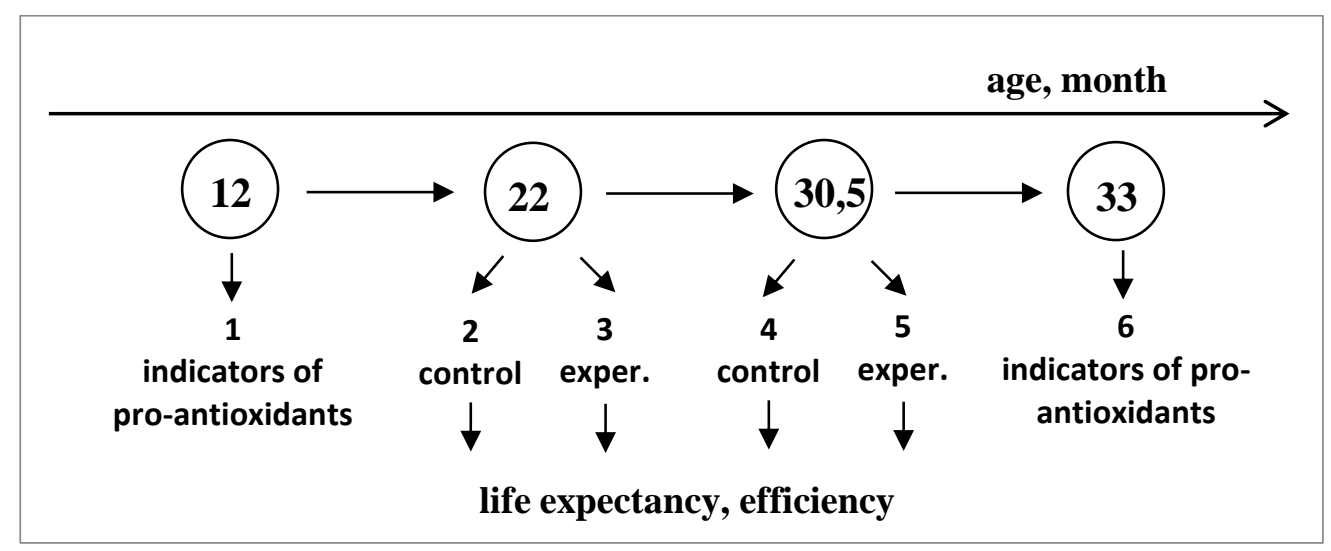

Figure 1. Scheme of the experiment. In 12 months old and 33 months old of animals (1 and 6 experimental groups), biochemical parameters were studied. From 22 months of animals, the 2nd - (control) group and the 3rd (experimental, which received a mixing factor with drinking water at a dose of $0.05-0.06 \mathrm{mg} / 100 \mathrm{~g}$ of body weight) were formed. From animals 30.5 months of age, two groups were also formed - 4th (control) and 5th (experimental), in which the life expectancy and working capacity were determined. 
Mix-factor is a food additive and obtained according to TUU 10.8 - 33206207 - 002: 2018. This is a natural complex obtained by cultivating Pleurotus ostreatus mushrooms in an aqueous culture with subsequent purification of fungal exometabolites. The composition of which consisted of: $67.2 \%$ oligosaccharides, free amino acids (trionine - $3 \%$, valine - $23 \%$, cysteine - $16 \%$, methionine $-11 \%$, leucine $-10 \%$, arginine - $4 \%$, isoleucine $-4 \%$, lysine $-1 \%$, the rest in trace amounts) and oligopeptides $-23.3 \%$, lipids $-11.5 \%$ and a small amount of vitamins (B1 - $0.38 \mathrm{mg} / \mathrm{l}$, B2 - $3.24 \mathrm{mg} / \mathrm{l}$, PP - 8, $3 \mathrm{mg} / \mathrm{l}$ ). All components are highly soluble in water, the solution is stable for at least 2 years. The mixing factor is non-toxic and had an immunomodulatory effect [16], a hepatotropic effect [16], and eliminated the toxic effect of copper sulfate [13].

In long-term experiments, adult animals (average weight 320 - $350 \mathrm{~g}$ ) took the mixing factor with drinking water. Drinking bowls with a mixing factor were changed daily, each rat drank about $20 \mathrm{ml}$ of water, which contained $160 \mu \mathrm{l}$, i. e. the dose of the mixing factor was $50 \mu \mathrm{l} / 100 \mathrm{~g}$ per day. During the experiment, body weight and body temperature (which were monitored weekly) did not change. No obvious pathologies were found in experimental rats.

\section{Determination of physiological parameters}

The bodyweight of the control and experimental animals was determined daily from 8 to 9:00 am before feeding [18].

The survival of the rats was calculated by the Kaplan-Meier method, and the comparison of the survival curves was performed by the Gehan method with Yates' correction. The results were considered significantly different at $\mathrm{p}<0.05$ between the control and research groups.

\section{Determination of health}

The performance of the animals was assessed by the time of running on a treadmill until exhaustion, at a belt speed of $40 \mathrm{~m} / \mathrm{sec}$. Animals for 22 months were divided into two groups: control and experimental, with 10 animals per group (Fig. 1). Before the start of the experiment, the maximum running time of the animals was determined, which was taken as $100 \%$ - the initial level. After that, the animals of both groups underwent training for running with the same load, which was $10 \%$ of the initial for each animal, every other day for 14 days, after which the maximum possible running time for each animal was again determined and this time was expressed as a percentage of the initial one, those the ability of the animals to train was determined.

\section{Determination of the content of lipid hydroperoxides}

The content of lipid hydroperoxide (HPL) in liver mitochondria was measured according to the Ohkawa method [19] and in serum according to the Asakawa method [20]. The absorption spectrum of the colored product was recorded on a Specord UV VIS two-beam spectrophotometer, measuring the difference in extinction at $535 \mathrm{~nm}$ and $520 \mathrm{~nm}$ [21]. The HPL content was expressed in equivalent amounts of MDA using a molar extinction coefficient of 1.56 × $105 \mathrm{M}-1$ x cm-1.

\section{Determination of enzyme activity}

Glutathione peroxidase activity (GP, EC 1.11.1.9) was determined in the cytosol fraction, liver mitochondria, and blood serum spectrophotometrically at $340 \mathrm{~nm}$ by the method of [22] in $50 \mathrm{mM} \mathrm{K}+, \mathrm{Na}+$ phosphate buffer ( $\mathrm{pH}$ 7.4) containing $1 \mathrm{mM}$ EDTA, 0, $15 \mathrm{mM} \mathrm{NADPH}, 1 \mathrm{U}$ of yeast glutathione reductase, $0.2 \%$ Triton $\mathrm{X}-100$, and $3 \mathrm{mM}$ Na azide to inhibit CAT. Cumene hydroperoxide was added at a concentration of $1.2 \mathrm{mM}$, hydrogen peroxide $-0.4 \mathrm{mmol}$. The incubation temperature was $-37^{\circ} \mathrm{C}$. The enzyme activity was expressed in nmol NADPH / min per $\mathrm{mg}$ of protein or $\mathrm{ml}$ of blood serum, taking into account the molar extinction coefficient of $6.22 \times 103 \mathrm{M}-1 \times \mathrm{cm}-1$.

Glutathione reductase activity (GR, EC 1.6.4.2;) in liver mitochondria and post-mitochondrial fraction of the liver, NADPH was measured spectrophotometrically in descending order of NADPH in a medium containing $50 \mathrm{mM} \mathrm{K}$ + -phosphate buffer, pH 7.4, 1 mM EDTA, $0.16 \mathrm{mM} \mathrm{NADPH}, 1 \mathrm{mM} \mathrm{GSSG}, 0.2 \%$ Triton X100 [23]. Incubation temperature - 37 0C. The enzyme activities were expressed in nmol NADPH / min mg protein, taking into account the molar extinction coefficient of $6.22 \times 103 \mathrm{M}-1 \times \mathrm{cm}-1$.

Malate dehydrogenase activity (MDG, EC 1.1.1.40;) was determined in liver mitochondria and postmitochondrial fraction spectrophotometrically by the rate of NADP + reduction [24] in $68 \mathrm{mM}$ Tris- $\mathrm{HCl}$ buffer, pH 7.4, containing $0.85 \mathrm{mmol} \mathrm{MnCl2,} 2 \mathrm{mM}$ malate, $0,4 \mathrm{mmol} \mathrm{NADP}+, 0.2 \%$ Triton X-100. Incubation temperature - 37 0C. Enzyme activities were expressed in nmol NADPH / min $\mathrm{mg}$ protein. 
Determination of glucose-6-phosphate dehydrogenase activity (EC 1.1.1.49) was carried out in mitochondria and postmitochondrial fraction of the liver spectrophotometrically according to the rate of $\mathrm{NADP}+$ reduction [25], in $120 \mathrm{mM}$ Tris- $\mathrm{HCl}$ buffer, $\mathrm{pH}$ 7.4, containing $10 \mathrm{mM} \mathrm{MgCl2,} 0.9 \mathrm{mM}$ NADP +, 0, 6 mmol 6 phosphogluconate, $0.2 \%$ Triton X-100. G6PDH activity was determined by subtracting 6FGDG activity from the total (6FGDG + G6PDH) activity, which was measured in the described medium supplemented with $2 \mathrm{mM}$ glucose-6-phosphate. Incubation temperature - 37 0C. The activity was expressed in nmol NADPH / min mg protein taking into account the molar extinction coefficient $6.22 \times 103 \mathrm{M}-1 \mathrm{~cm}-1$

Isocitrate dehydrogenase activity (ICDG, EC 1.1.1.42) in liver mitochondria and postmitochondrial fraction was determined spectrophotometrically by the rate of NADP + reduction [26] in $34 \mathrm{mM}$ Tris- $\mathrm{HCl}$ buffer, pH 7.4, containing $0.34 \mathrm{mmol}$ EDTA, $1.5 \mathrm{mM} \mathrm{MnCI} 2,0.1 \mathrm{mmol} \mathrm{NADP}+, 1.5 \mathrm{mmol}$ isocitrates, $0.2 \%$ Triton X-100. Temperature $-37^{\circ} \mathrm{C}$. Activity was expressed in nmol NADPH / min mg protein.

Determination of the antioxidant activity of the mixing factor in the in vitro model system was carried out as described in [27], and the antiradical activity of the mixing factor as described in [28].

\section{Statistical analysis of results}

The results obtained were processed statistically using the Statistika V.6 software package. The significance of differences between groups was assessed using the method of nonparametric statistics (Wilcoxon-Mann-Whitney test). The survival curves of rats were calculated by the Kaplan-Meier method, and the comparison of the survival curves was carried out according to the Gehan method with the Yates correction. Differences were considered statistically significant at $\mathrm{p}<0.05$, which were denoted in graphs and tables by the symbol "*".

\section{Results and discussion}

The influence of the mixing factor on some indicators of the pro-antioxidant system when used from 22 months until the end of life

The number of lipid hydroperoxides in liver mitochondria in 33 months old rats increased by $25 \%$ (Fig. 2A) compared with 12 months old animals when the animals were kept under standard vivarium conditions. The content of lipid hydroperoxides in these animals in the blood serum can serve as an indicator of the integral characteristics of free radical processes in the body. It turned out that the content of lipid hydroperoxides in the blood serum was 33 months old animals were $41 \%$ more compared with 12 months old animals (Fig.2B).

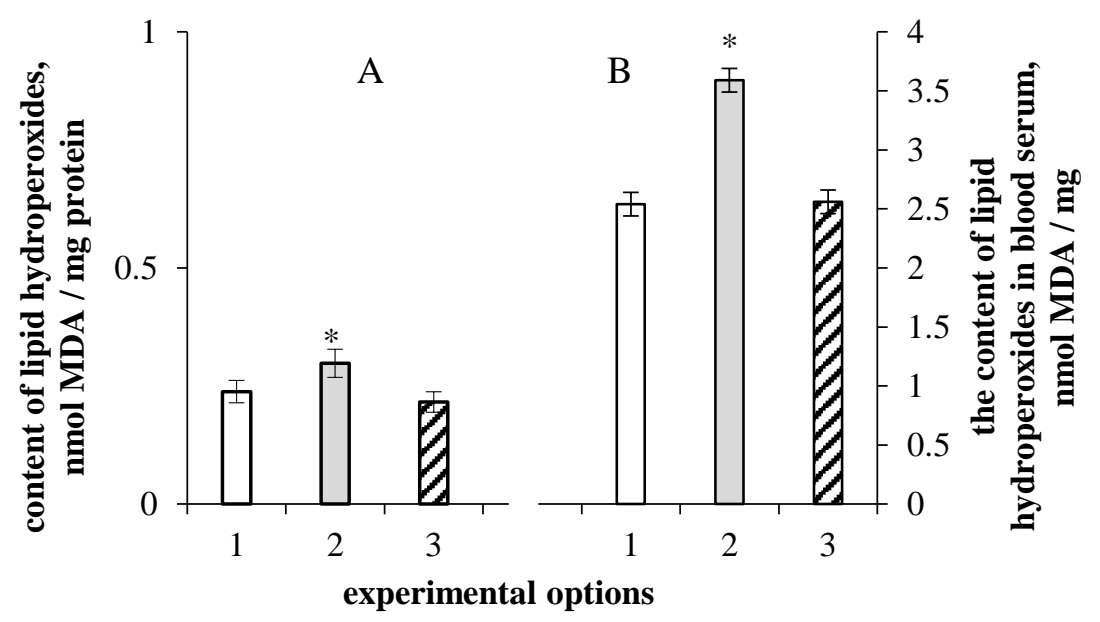

Figure 2. Content of lipid hydroperoxides in the liver mitochondrial fraction (A) and blood serum (B) at 12 months old animals (1) and 33 months old animals (2) that were kept under standard conditions and animals that received the mixing factor daily with drinking water, starting from 22 months of age, at a dose of $0.05-0.06 \mathrm{mg} / 100 \mathrm{~g}$ of body weight (3)

The average values for 6 animals in each variant are presented,

* - differences are indicated in comparison with the intact control 12 months of age 
Therefore, from 12 to 33 months old in animals kept under standard vivarium conditions, the content of products of free radical reactions increased. There is a well-pronounced relationship between the content of lipid hydroperoxides in liver mitochondria and the body as a whole, which makes it possible to use liver mitochondria as a "marker" of age-dependent changes in the body.

In the event that animals, starting from 22 months old age, the mixing factor was taken daily with drinking water at a dose of $0.05-0.06 \mathrm{mg} / 100 \mathrm{~g}$ of body weight, then the content of lipid hydroperoxides both in mitochondria and in blood serum did not differ from the content at 12 months old intact animals (Fig. 2A, B).

The process of maintaining the number of lipid hydroperoxides at a stable level can be implemented in two ways. Firstly, the components of the mixing factor can activate the antioxidant defense system of the adult organism, and secondly, the components of the mixing factor themselves can act as antioxidants and prevent the formation of products of free radical reactions, and, possibly, have a polyfunctional effect.

It was previously shown that glutathione peroxidase is one of the antioxidant enzymes of the glutathione cycle, which actively responds to various exogenous influences [15].

It turned out that the activity of glutathione peroxidase in liver mitochondria for 33 months old rats were $25 \%$ less active compared to 12 months old animals under standard conditions of their keeping (Fig. 3A). In the event that animals, starting from 22 months old, the mixing factor was received daily with drinking water, then its activity in 33 months old of animals in all studies of erythrocyte and blood serum fractions did not differ from 12 months old intact animals (Fig. 3A).

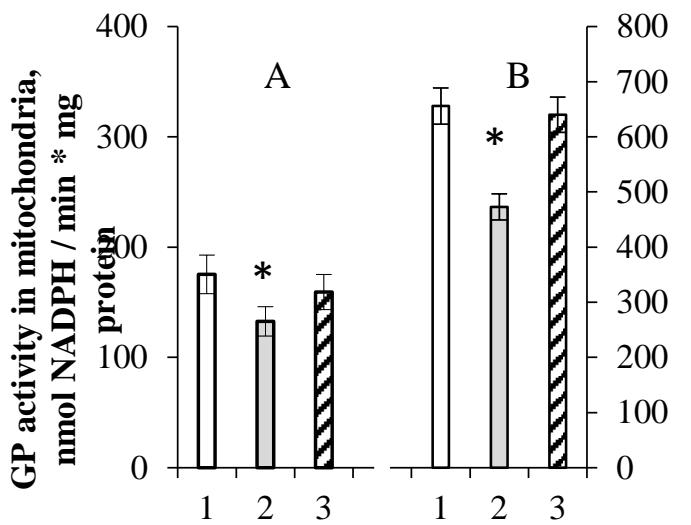

experimental options
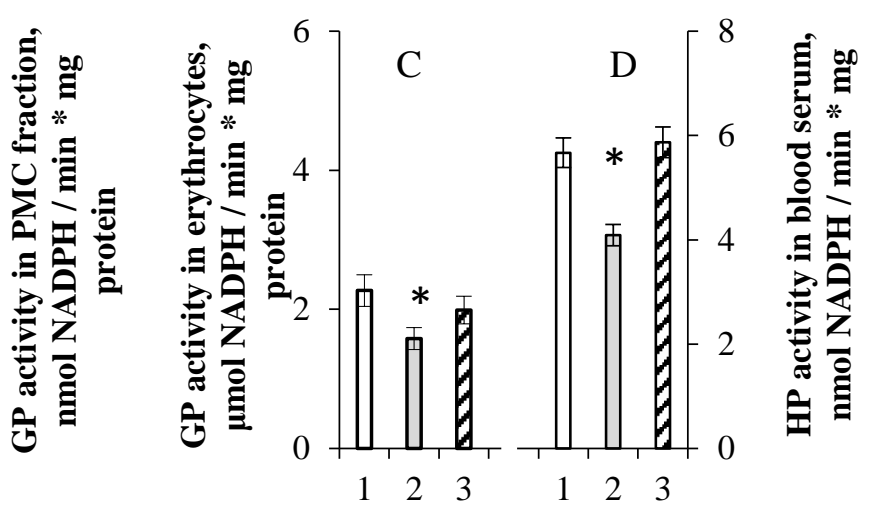

experimental options

Figure 3. Activity of glutathione peroxidase in the fraction of liver mitochondria (A), postmitochondrial fraction (B), erythrocytes (C), and blood serum (D) at 12 months old animals (1) and 33 months old animals (2) that were kept under standard conditions and animals that received daily mixing factor with drinking water, starting from 22 months of age, at a dose of $0.05-0.06 \mathrm{mg} / 100 \mathrm{~g}$ of body weight (3)

The average values for 6 animals in each variant are presented,

* - the differences are indicated in comparison with the intact control 12 months of age

Currently, 7 isoforms of glutathione peroxidase have been isolated, which have different tissue and cellular localization [29]. Sufficiently high activity of glutathione peroxidase was detected in the postmitochondrial fraction of the liver. This can be explained by the fact that this fraction contains at least two isoforms of this enzyme: microsomal and cytosolic.

Consequently, the components of the mixing factor activate various isoforms of glutathione peroxidase localized in various compartments of liver cells, erythrocytes, and blood serum.

Consequently, maintaining the level of lipid hydroperoxides at 33 months old animals at the level of 12 months old animals were provided with an increase in the activity of glutathione peroxidase against the background of the introduction of a mixing factor.

It was of interest to study the activity of other antioxidant enzymes. In this regard, the activity of glutathione reductase, glucose-6-phosphate dehydrogenase, isocitrate dehydrogenase, and malate dehydrogenase in mitochondria and the post-mitochondrial fraction of the liver was investigated at 12 and 33 months old animals. 
It turned out that glutathione reductase activity in rat liver mitochondria from 12 to 33 months old age remained unchanged (Fig. 4A).
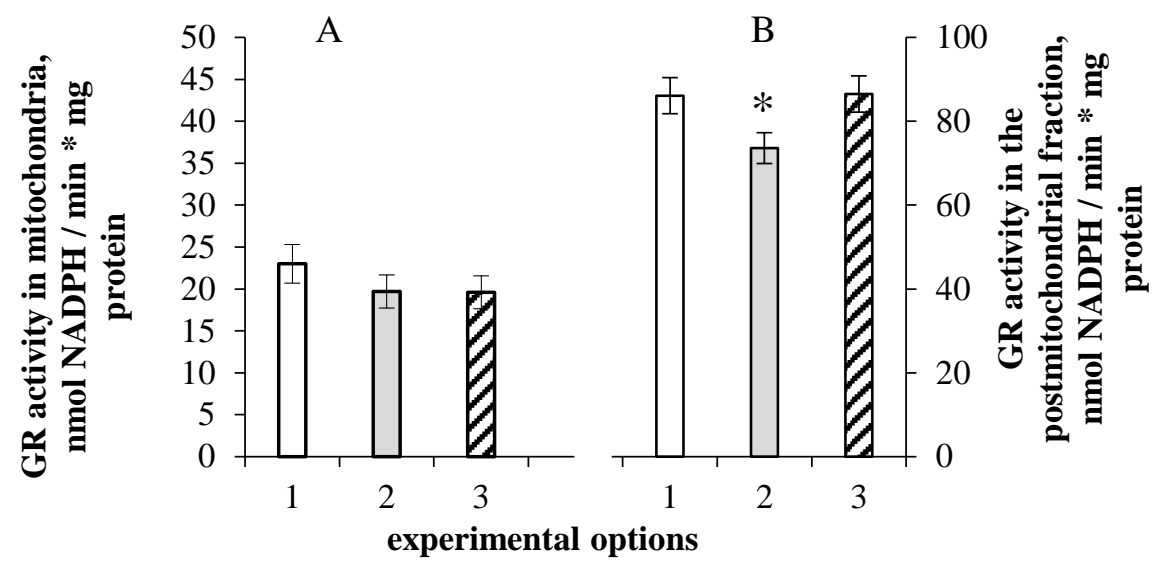

Figure 4. Activity of glutathione reductase in the fraction of liver mitochondria (A) and a postmitochondrial fraction (B) at 12 months old animals (1) and 33 months old animals (2) that were kept under standard conditions and animals that received daily mixing factor daily with drinking water, starting from 22 months of age (3), at a dose of $0.05-0.06 \mathrm{mg} / 100 \mathrm{~g}$ of body weight $(\mathrm{n}=6)$. The average values for 6 animals in each variant are presented,

* - the differences are indicated in comparison with the intact control 12 months of age

At the same time, in the postmitochondrial fraction, its activity significantly decreased by $25 \%$ at 33 months old animals compared with 12 months old animals and long-term administration of the mixing factor led to its increase up to the level of 12 months old animals (Fig.4B).

Activities of glucose-6-phosphate dehydrogenase, isocitrate dehydrogenase, and malate dehydrogenase at 33 months old animals did not differ from that for 12 months old (table).

Table

Enzyme activity in mitochondria and a postmitochondrial fraction of rat liver for 12 months and 33 months of, which were kept under standard conditions, and animals that received the mixing factor daily with drinking water, starting from 22 months of age, at a dose of $0.05-0.06 \mathrm{mg} / 100 \mathrm{~g}$ of body weight until the end of life (for 33-month-old animals, the numerator is animals that did not receive the mixing factor (control), the denominator is the animals that received the mixing factor)

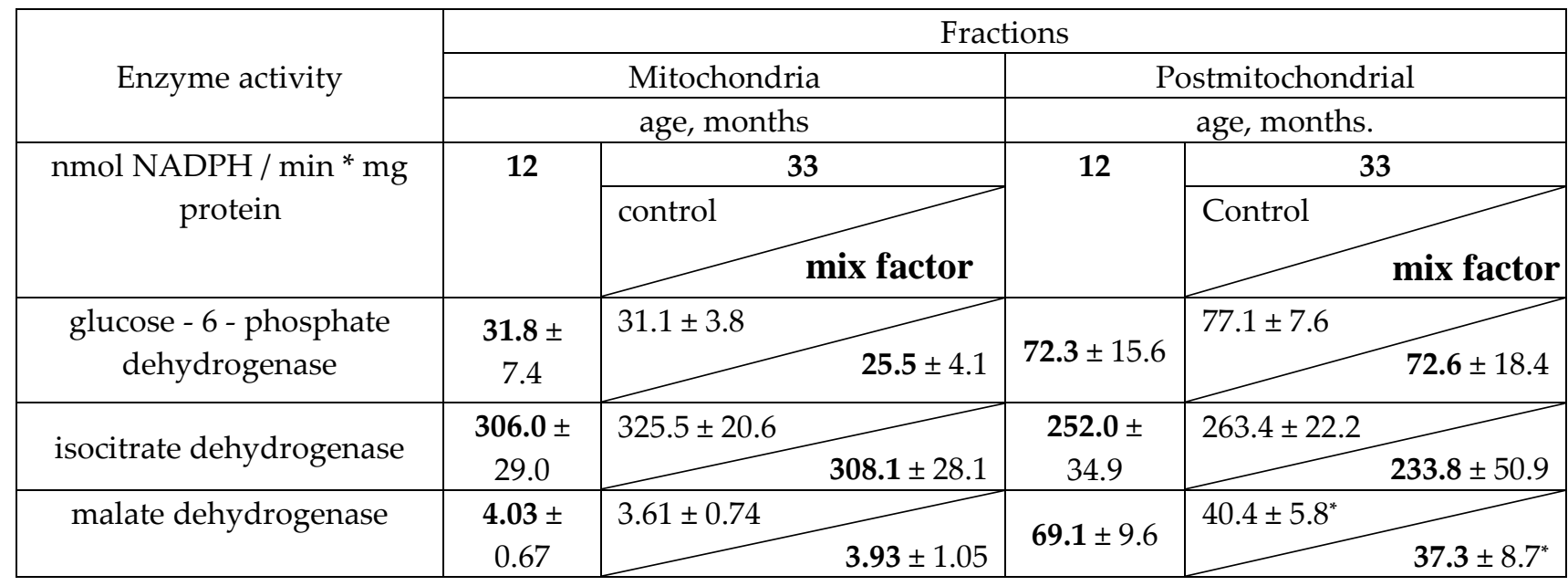

Notes: ${ }^{*}-p<0.05$

In the next series of experiments, the antiradical and antioxidant properties of colostrum components were determined in an in vitro system.

It was found that the addition of a mixing factor to the model system at a concentration of $2.5 \mu \mathrm{l}$ per 1 $\mathrm{ml}$ of medium slowed down the oxidation of yolk lipoproteins by $28 \%$, and with an increase in concentration to 5 and $10 \mu \mathrm{l}$, this effect linearly increased (Fig. 5). If we compare the antioxidant activity of the mixing factor 
with quercetin (a flavonoid with a known antioxidant activity [30], then the mixing factor was not inferior to it in this indicator.

The components of the mixing factor also had a pronounced effect of interception of $\mathrm{OH}$ - radicals in the model system. Thus, at a concentration of $2.5 \mu \mathrm{g}$ per $1 \mathrm{ml}$, it "intercepted" up to $43 \% \mathrm{OH}$ - radicals, and at a concentration of $10 \mu \mathrm{l}$ per $1 \mathrm{ml}$ - up to $74.6 \%$, induced in the model system of radicals (Fig. 5).

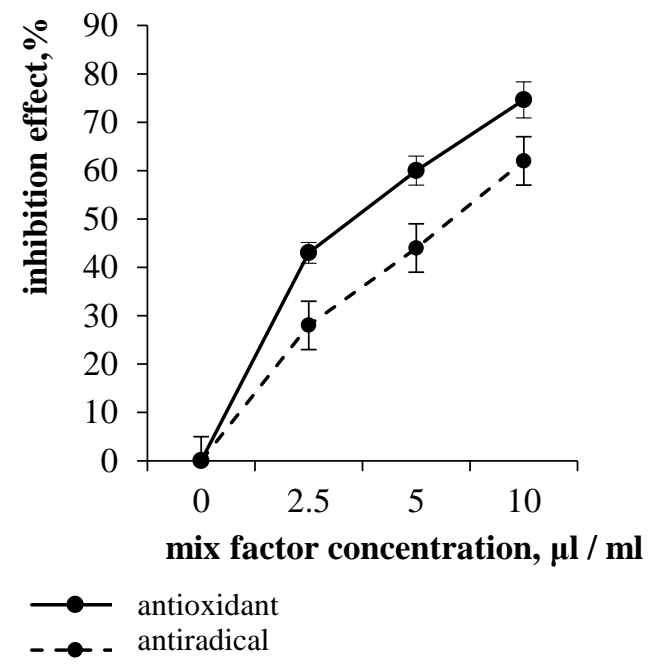

Figure 5. Antioxidant and antiradical activity of the mixing factor in the in vitro system as a percentage of the control values

Consequently, the components of the mixing factor themselves have antioxidant activity in the model system.

The effect of the mixing factor on life expectancy

From animals of 22 months of age, two experimental groups were formed: control (31 animals) and experimental (30 animals), which received daily mixing factor with drinking water, on average, $0.05 \mathrm{ml}$ per $100 \mathrm{~g}$ of body weight until the end life (Fig. 1).

Long-term administration of the mixing factor from 22 months of age slowed down the death of animals in the period from 740 days (25.6 months) to 860 days (28.6 months) of observations (Fig.6A). However, starting from day 940 (31.3 months), the control and experimental groups did not differ in terms of life expectancy (Fig. 6A). The maximum life expectancy (death of the last animal) came on 1100 days (36.6 months). 


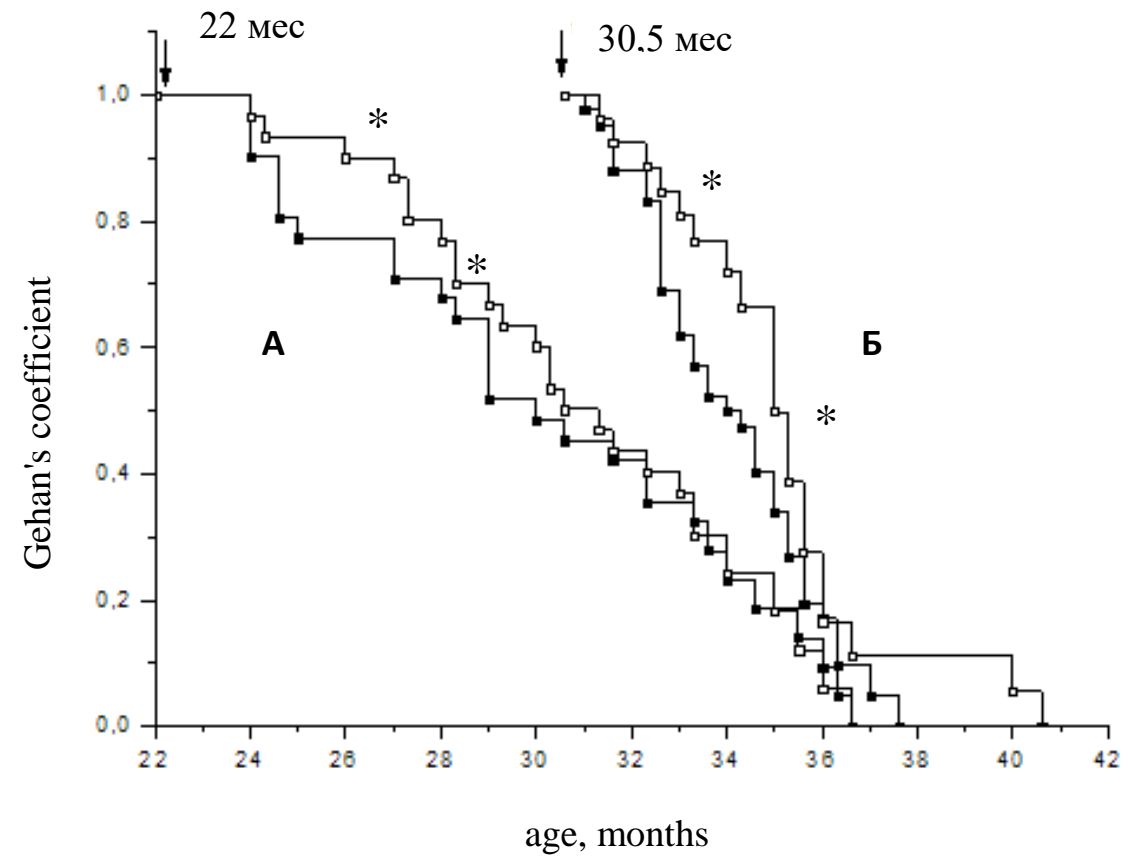

Figure 6. Survival curves for the control group of animals () and animals that received daily mixing factor () from 22 months of age until the end of life (A) and received the mixing factor from 30.6 months of age until the end of life (B).

* - marked the initial and final significant differences in comparison with the control

It can be assumed that the effect of the mixing factor on the organism will depend not only on the dose and its characteristics but also on the functional characteristics of the organism itself at this time [31 7]. In this regard, in the second series of experiments, two groups were also formed: control (40 rats) and experimental (26 rats) from animals at the age of 920 days (30.6 months of age). The experimental group received the mixing factor daily with drinking water at the same dose $(0.05 \mathrm{ml} / 100 \mathrm{~g}$ of body weight) until the end of life.

The fact was confirmed that the control group of animals had the same maximum life span of 1100 days, as in the first series of experiments (Fig. 6B). At the same time, the survival curve in the experimental group differed from the control in that in some animals, natural death occurred much later, and some animals survived up to 1220 days (40.6 months) (Fig. 6B).

Consequently, the mixing factor had a more pronounced effect on increasing life expectancy if it was used at the later stages of ontogenesis.

As you know, the main position of the paradigm of gerontology boils down to the fact that with increasing age, the ability of animals to adequately respond to numerous exogenous factors decreases, i.e. the ability to adapt decreases [32]. Perhaps the most pronounced effects of age-related changes were manifested in the loss of the ability to endure physical activity.

If the components of the mixing factor improve the quality of life of old animals, then we can expect that they will also affect the ability of such animals to adapt to stress loads, in particular, the ability to do work. 

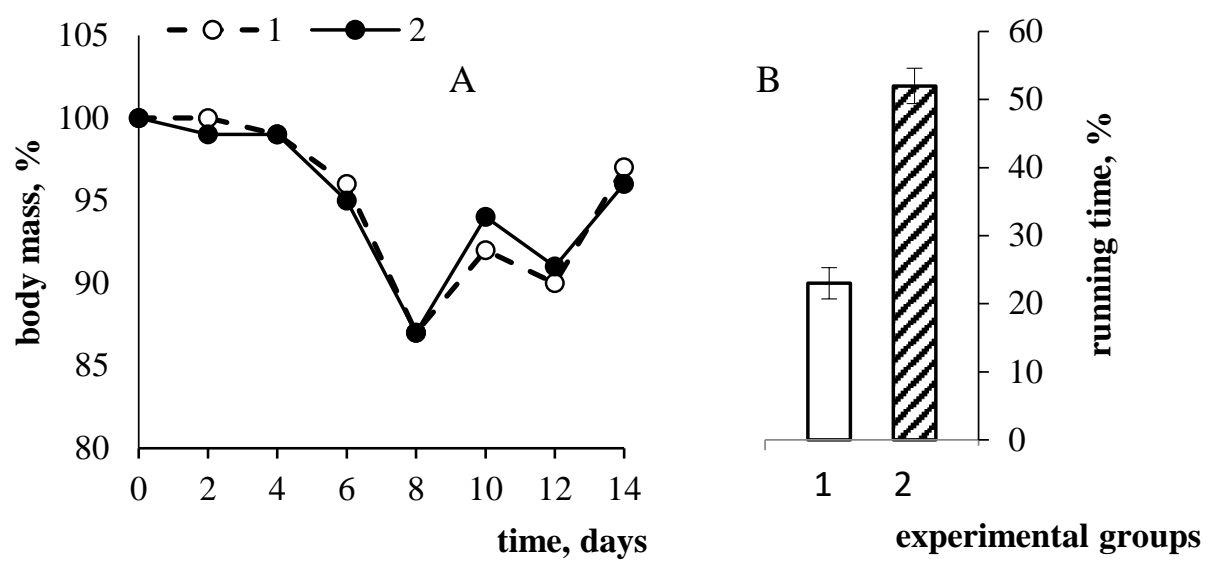

Figure 7. Change in body weight from the initial (A) in animals of the control group (1) and animals receiving daily mixing factor during training in a treadmill for 14 days (2), an increase in running time in a treadmill after 14 days of training for animals in the control group (1) and the group that received the mixing factor (2) (B) from the original

To check this, the ability of 22 months of rats to perform the maximum possible physical activity was determined - running on a treadmill until exhaustion.

It turned out that such physical training was accompanied by a loss of body weight to the same extent in the control and experimental animals, which was especially pronounced on the 8th day of training, after which their body weight was restored, despite continued physical exertion (Fig. 7A).

The results indicate that the animals experienced stress and adapted well to it. In the control group, the running time increased by $22 \%$ compared to the first run, while in the group of animals receiving the mixing factor, this time increased by $55 \%$ from the initial one (Fig. 7B).

Consequently, the mixing factor improved the adaptive ability to physical activity of old animals and slowed down the process of their natural death for a relatively short period.

According to the United Nations, by 2025 the number of elderly people will reach 1 billion people, which will be $13.7 \%$ of the world's population [33]. The problem of improving the quality of life of older people, in particular, their ability to perform physical work, is the most important medical and social problem. Although the data obtained in this work do not yet provide grounds for attributing the mixing factor to geroprotectors, it deserves attention as a factor influencing the performance and adaptability of the organism to stress factors. Earlier, on the model of intoxication of the body with copper ions, which was accompanied by the development of liver fibrosis, it was shown that the mixing factor increased the ability to do work in such animals and eliminated several biochemical parameters that are characteristic of liver fibrosis [13].

Long-term intake of the mixing factor in old animals (22 months) ensured the maintenance of the number of lipid hydroperoxides in the blood serum at the level of 12 months old of animals, and this could be associated both with the activation of antioxidant enzymes - glutathione peroxidase and glutathione reductase and with the manifestation of the antioxidant components of the mixing factor themselves. properties. We cannot say that only the change in the pro-antioxidant system, which is caused by the action of the mixing factor, provided the old animals with a slight increase in life expectancy and their ability to do work. We believe that the pro-antioxidant system, as a self-regulating system, is the leading (basic) system of regulation of many-body systems. It is known that phagocytic cells use the oxidative burst to destroy the bacteria captured by them; the function of the body's redox system is closely related to the activity of the immune system [34,35].

We have previously shown that the mixing factor activated the cellular link of immunity in liver fibrosis [16].

The determining factor in the regulation of the redox system itself is the content of reactive oxygen species (ROS) [36,37]. It follows from this that an increase or decrease in ROS for some reason will shift the equilibrium towards pro- or antioxidants. This classical principle of regulation ensures the maintenance of many, if not all, systems of regulation. However, it remains unclear the rules by which such a principle of implementation functions, i.e. It remains unclear who and how determines going beyond homeostasis, 
whether it is possible to ensure the long-term preservation of homeostasis at the later stages of ontogenesis, and how this can be achieved.

In this regard, it is necessary to pay attention to the fact that the mixing factor had a regulatory effect on those indicators of the redox system that "went" beyond the "previous states". So, if the previous states are the characteristics of the redox system of 12 months old of animals, then an increase in the content of lipid hydroperoxides in 33 months old of animals is a conditional exit from the previous states. The components of the mixing factor retained these parameters in 33 months old of animals within the previous limits. At the same time, the mixing factor did not affect glucose-6-phosphate dehydrogenase, malate - isocitrate dehydrogenase, which remained unchanged in 33 months old of animals compared to 12 months old of animals. In addition, the mixing factor had a more pronounced effect in increasing life expectancy when it was started to be given to very old animals (from 30.6 months of age), i.e. those in which homeostatic parameters are changed to a greater extent than in 22 months of age animals. One gets the impression that the components of the mixing factor ensure the maintenance of the indicators of the redox system in the body in the limit of the previous states, i.e. they "ensure" the observance of some rules for the functioning of autoregulation systems.

The mixing factor is a multi-component natural complex and, depending on the dose and characteristics of the functional state of the organism itself, it can act as both a prooxidant and an antioxidant. This was shown using the example of low molecular weight components of colostrum, which caused similar physiological effects as the mixing factor [12]. It can be assumed that the components of the mixing factor affect not only the indicators of the pro-antioxidant system but also regulate the functioning of the immune system, which ensures the autoregulation of altered homeostatic indicators. We believe that these effects are regulated by mechanisms similar to the hormesis phenomenon. This is evidenced by the data we obtained earlier on the model of the induction of the hormesis effect on the toxic effects of copper sulfate [13].

Of course, this speculative hypothesis has no experimental confirmation, but there is no doubt that the mixing factor is involved in the regulation of the functions of the body's redox system.

It is important to note the peculiarities of the temporal characteristics of the survival curves in animals treated with the mixing factor. The shift of the survival curves in the case of taking the mixing factor from 22 months of age to the right took place in the period from 25-30 months of age. In the case of administration of the mixing factor from 30.5 months of age, the effect of increasing life expectancy was manifested from 33 to 36 months of life, and the last animals died at 41 months, and not at 37.5 months, as in the control variant. Consequently, the effect of delayed mortality was relatively short-lived, which makes it possible to attribute it to the phenomenon of hormesis, i.e. nonspecific stimulation of the body's resistance.

\section{Conclusions}

1. In the period from 12 to 33 months of age, an increase in the content of lipid hydroperoxides in the body was observed against the background of a decrease in the activity of glutathione peroxidase and glutathione reductase;

2. taking a biologically active additive - "mixing factor", starting from 22 months of age (old animals) ensured the preservation of the indicators of the pro-antioxidant system at the level of 12 months old (adult) animals;

3. taking the mixing factor from 30.5 months of age caused a more pronounced effect of increasing life expectancy compared to taking it from 22 months of age;

4. the increase in life expectancy against the background of intake of the mixing factor is due to the regulatory influence of the components of the mixing factor not only on the redox system of the body but also on other regulatory systems, in particular, the immune system, which explains the increase in resistance to physical activity in old animals.

Author Contributions: All authors participated equally in writing this article.

Conflicts of Interest: The authors declare no conflict of interest. 


\section{Information about Authors:}

Anatoly I. Bozhkov - DSc (Biology), Prof. of the V.N. Karazin Kharkov National University, Director of the Research Institute of Biology; https://orcid.org/0000-0001-8418-5716

Ievgeniy G. Ivanov - PhD (Biology), Research Institute of Biology of the V.N. Karazin Kharkov National University; https://orcid.org/0000-0001-5146-2705

Elena M. Klimova - DSc (Biology), Head of the diagnostic laboratory of enzyme-linked immunosorbent assay and immunofluorescence analysis of the V. T. Zaitsev Institute of General and Emergency Surgery of the National Academy of Medical Sciences of Ukraine

Natalia I. Kurguzova - Senior Lecturer of the V.N. Karazin Kharkov National University

Andrii A. Bozhkov - Junior Researcher Research Institute of Biology of the V.N. Karazin Kharkov National University Anatoly V. Goltvyanskiy - PhD (Biology), Associate Professor of the Department of Molecular Biology and Biotechnology of the V.N. Karazin Kharkov National University; https:/orcid.org/0000-0003-3484-8053

Yuri V. Nikitchenko - DSc (Biology), Research Institute of Biology of the V.N. Karazin Kharkov National University 


\section{References}

1. Anisimov, V. N. Means for the prevention of premature aging (geroprotectors). Advances in gerontology 2000, 4, 275-277. (in Russian)

2. Gonzalez-Freire, M.; De Cabo, R.; Bernier M., Solott, S. J; Fabbry, E. et al. Reconsidering the role of mitochondria in aging. Journals of Gerontology Series.A 2015, 70, 1334-1342. https://doi.org/10.1093/gerona/glv070

3. Jones, D. P. Redox theory of aging. Redox biol 2015, 5, 71-79. https://dx.doi.org/10.1016\%2Fj.redox.2015.03.004

4. Sohal, R. S. The free radical hypothesis of aging: an appraisal of the current status. Aging Clin Exp Res 1993, 5, 3-17. https://doi.org/10.1007/BF03324120

5. Ziegler, D. V.; Wiley, C. D.; Velarde, M. C. Mitochondrial effectors of cellular senescence: beyond the free radical theory of aging. Aging cell 2015, 14, 1-7. https://doi.org/10.1111/acel.12287

6. Mittler, R. ROS are good. Trends Plant Sci 2017, 22, 11-19. https://doi.org/10.1016/j.tplants.2016.08.002

7. Surai, P. F. Antioxidant systems in poultry biology: superoxide dismutase. J Anim Res Nutr 2016, 1, p 8. https://doi.org/10.21767/2572-5459.100008

8. Vilhardt, F.; Haslund-Vinding, J.; Jaquet, V.; McBean, G. Microglia antioxidant systems and redox signalling. Br J Pharmacol 2017, 174, 1719-1732. https://doi.org/10.1111/bph.13426

9. Zhang, H.; Tsao, R. Dietary polyphenols, oxidative stress and antioxidant and anti-inflammatory effects. Curr. Opin. Food Sci. 2016, 8, 33-42. https://doi.org/10.1016/j.cofs.2016.02.002

10. Halliwell, B.; Gutteridge, J. M. Free radicals in biology and medicine. Oxford University Press: Oxford, 2015. https://doi.org/10.1093/acprof:oso/9780198717478.001.0001

11. Winterbourn, C. C. Are free radicals involved in thiol-based redox signaling? Free Radic. Biol. Med. 2015, 80, 164-170. https://doi.org/10.1016/j.freeradbiomed.2014.08.017

12. Bozhkov, A. I.; Ivanov, E. G.; Mohammad, A.Y.; Al Begai; Mohammad, M.A. et al. Low-molecular weight cow colostrum components in functional nutrition. J Am Coll Nutr 2017, 6, 11-17. http://dx.doi.org/10.6000/1929-5634.2017.06.01.2

13. Bozhkov, A. I.; Nikitchenko, Y. V.; Lebid, K. M.; Ivanov, E. G.; Kurguzova, N. I. et al. Low molecular weight components from various sources eliminate oxidative stress and restore physiological characteristic of animals at early stages of cu-induced liver fibrosis development. Transl Biomed 2017, 8, 2. http://dx.doi.org/10.2167/2172-0479.1000107

14. Bozhkov, A.; Nikitchenko, Y.; Lebed', K.; Linkevych, O.; Kurguzova, N. et al. The cyclic feeding regime induces decaying age-dependent oxidative stress and regulates the cell chain of the immunity. Advances in Aging Research 2016, 5, 151-155. http://dx.doi.org/10.4236/aar.2016.56015

15. Kurguzova, N. I.; Bozhkov, A. I.; Nikitchenko, Y. V.; Al Begai, M. A. Y.; Goltvyansky, A. V. et al. Interconnection of antitoxic and antioxidant systems of the organism under the action of natural low molecular complex-fungidol. American Journal of Biomedical and Life Sciences 2015, 2, 25-32. http://dx.doi.org/10.11648/j.ajbls.s.2014020601.15

16. Bozhkov, A. I.; Linkevych, O. S.; Ivanov, E. G.; Klimova, O. M.; AlBegai, M. A. Y. et al. Low molecular weight components of colostrum regulate the activity of cellular component of the immune system in animals with Cu-induced liver fibrosis. International Journal of Current Research 2016, 8, 44129-44137.

17. Bozhkov, A. I.; Klimova, O. M.; Nikitchenko, Y. V.; Kurguzova N. I.; Linkevych, O. S. et al. Ontogenetic Approach to the Study of Mechanisms of Copper-Induced Liver Fibrosis. Advances in Aging Research, 2017, 6, p 39. http://dx.doi.org/10.4236/aar.2017.63005

18. Klimova, E. M.; Merezhko, O. S.; Al-Bahadly Ali, M. M.; Kurguzova, N. I.; Bozhkov, A. I. Age determines the intensity of thyrotropic hormone production in response to copper sulphate intoxication. Advances in Biology \& Earth Sciences 2018, 3, 234-240.

19. Ohkawa, H.; Ohahi, H. N.; Jadi, K. Assay for lipid peroxides in animal tissues by thiobarbituric acid reaction. Anal Biochem, 1979, 95, 351-358. https://doi.org/10.1016/0003-2697(79)90738-3

20. Asakawa, T.; Matsushita, S. Coloring condition of thiobarbituric acid test for detecting lipid hydroperoxides. Lipids 1980, 15, 137-140. https://doi.org/10.1007/BF02540959

21. Massie, H. R.; Aiello, V. R.; Banziger, V. Iron accumulation and lipid peroxidation in aging C57BL/6J mice. Exp. Gerontol. 1983, 18, 277-285. https://doi.org/10.1016/0531-5565(83)90038-4 
22. Paglia, D. E.; Valentine, W. N. Studies on the quantitative and qualitative characterization of erythrocyte glutathione peroxidase. J. Lab. Clin. Med. 1967, 70, 158-169.

23. Carlberg, I.; Mannerviek, B. Glutathione reductase levels in rat brain. J. Biol. Chem. 1975, 250, 54755480 .

24. Usatenko, M. S.; Tsoncheva, A. B. Effect of insulin deficit and hydrocortisone on the activity of NADPand NAD-dependent malate dehydrogenase in the liver and kidney cortex of rats. Medicinal chemistry issues 1974, 20, 401-406. (in Russian)

25. Zaheer, N.; Tewary, K. K.; Krishnan, P. S. Mitochondrial forms of glucose-6-phosphate dehydrogenase and 6-phosphogluconic dehydrogenase in rat liver. Arch Biochem Biophys 1967, 120, $22-34$. https://doi.org/10.1016/0003-9861(67)90593-0

26. Bauman, D. E.; Brown,R. E.; Davis, C. J. Pathways of fatty acid synthesis and reducing equivalent generation in mammary gland of rat sow and cow. Arch Biochem Biophys 1970, 140, 237-244. https://doi.org/10.1016/0003-9861(70)90028-7

27. Klebanov, G. I.; Babenkova, I. V.; Teselkin, J. O.; et al. Evaluation of antioxidantactivity of blood plasma using yolk. Laboratornoe delo 1988, 5, 59-62. (in Russian).

28. Halliwell, B.; Gutteridge, J. M. C.; Aruoma, O. I. The deoxyribosemethod: a simple "test-tube" assay for determination of rate constants of hydroxyl radical. Analitical Biochemistry 1987, 165, 215 - 219. https://doi.org/10.1016/0003-2697(87)90222-3

29. Maiorino, M.; Bosello-Travain, V.; Cozza, G.; Miotto, G.; Roveri, A. et al. Understanding mammalian glutathione peroxidase 7 in the light of its homologs // Free Radic Biol Med 2015, 83, 352-360. https://doi.org/10.1016/j.freeradbiomed.2015.02.017

30. Kostyuk, V. A.; Potapovich, A. I.; Kovaleva, Zh. V. Simple and sensitive method for determining the activity of superoxide dismutase, based on the oxidation reaction of quercetin. Medicinal chemistry issues 1990, 36, 88-91. (in Russian)

31. Bozhkov A. I., Kovaleva M. K., Menzyanova N. G. et al. The rate of aging of the Dunaliella viridis Teodor. culture depends on the algae pre-adaptation to copper sulfate toxic effect. Advances in Aging Research 2014, 3, 187-198. http://dx.doi.org/10.4236/aar.2014.32027

32. Wahl, H. W.; Becker, S.; Burmedi, D. The role of primary and secondary control in adaptation to agerelated vision loss: a study of older adults with macular degeneration. Psychology and Aging 2004, 19, 235 - 239. https://psycnet.apa.org/doi/10.1037/0882-7974.19.1.235

33. Kytikova, O. Yu.; Novgorodtsev, A. D.; Gvozdenko, T. A. Medical ozone-like redox-oxidative hormetin in geriatrics. Health. Medical ecology. The science 2014, 55, 26 - 30. (in Russian)

34. Bounous, G.; Molson, J. H. The antioxidant system. Anticancer Res 2003, 23, 1411-1415.

35. Nietzel, T.; Mostertz, J.; Hochgräfe, F.; Schwarzländer, M. Redox regulation of mitochondrial proteins and proteomes by cysteine thiol switches. Mitochondrion 2017, 33, 72-83. https://doi.org/10.1016/j.mito.2016.07.010

36. Grzesik, M.; Naparło, K.; Bartosz, G.; Sadowska-Bartosz, I. Antioxidant properties of catechins: Comparison with other antioxidants. Food Chem 2018, 241, 480-492. doi: 10.1016/j.foodchem.2017.08.117.

37. Halliwell, B. Reactive oxygen species in living systems: source, biochemistry, and role in human disease. Am J Med 1991, 91, 3C, 14S-22S. doi: 10.1016/0002-9343(91)90279-7 